\title{
Molecular dynamics in the energy sector: \\ Experiment and modeling of the $\mathrm{CO}_{2} / \mathrm{CH}_{4}$ mixture
}

\section{Supporting Information}

Martin R. Schenk ${ }^{* 1}$, Thorsten Köddermann ${ }^{1}$, Karl N. Kirschner, ${ }^{1}$ Sandra Knauer ${ }^{2}$, Dirk Reith ${ }^{1}$

${ }^{1}$ Bonn-Rhein-Sieg University of Applied Sciences, Institute of Technology, Resource and

Energy-Efficient Engineering (TREE), Grantham-Allee 20, D-53757 Sankt Augustin, Germany.

${ }^{2}$ Eurotechnica GmbH, An den Stuecken 55, D-22941 Bargteheide, Germany

*Corresponding authors email address: martin.r.schenk@gmail.com 
Table S1: Surface tension of pure $\mathrm{CO}_{2}$ at various temperatures and corresponding equilibrium pressures, experimental results from the present work. The uncertainties are the ranges of 6 independent measurements at each data point.

\begin{tabular}{|l|l|l|l|l|}
\hline$T / \mathrm{K}$ & $p / \mathrm{MPa}$ & $\gamma / \mathrm{mN} / \mathrm{m}$ & $\Delta \gamma / \mathrm{mN} / \mathrm{m}$ & $\Delta \gamma_{\text {rel }} / \%$ \\
\hline 239 & 1.2 & 12.4 & 1.9 & 15.3 \\
\hline 257 & 2.2 & 8.3 & 1.1 & 13.3 \\
\hline 265 & 2.8 & 7.0 & 0.7 & 10.0 \\
\hline 269 & 3.1 & 5.6 & 0.7 & 12.5 \\
\hline 273 & 3.5 & 4.7 & 0.2 & 4.2 \\
\hline
\end{tabular}

Table S2: Surface tension of the $\mathrm{CO}_{2} / \mathrm{CH}_{4}$ mixture at various pressures and temperatures of $223 \mathrm{~K}$ and $233 \mathrm{~K}$, experimental results from the present work. The uncertainties are the ranges of 6 independent measurements at each data point.

\begin{tabular}{|l|l|l|l|l|}
\hline$T / \mathrm{K}$ & $p / \mathrm{MPa}$ & $\gamma / \mathrm{mN} / \mathrm{m}$ & $\Delta \gamma / \mathrm{mN} / \mathrm{m}$ & $\Delta \gamma_{\text {rel }} / \%$ \\
\hline 223 & 3.0 & 8.7 & 1.0 & 11.5 \\
\cline { 2 - 6 } & 3.9 & 6.0 & 0.4 & 6.7 \\
\cline { 2 - 6 } & 5.5 & 3.0 & 0.7 & 23.3 \\
\hline 233 & 3.0 & 9.4 & 1.1 & 11.7 \\
\cline { 2 - 6 } & 4.1 & 6.1 & 0.7 & 11.5 \\
\cline { 2 - 5 } & 5.0 & 4.6 & 0.5 & 10.9 \\
\cline { 2 - 5 } & 5.1 & 3.6 & 0.1 & 2.8 \\
\hline
\end{tabular}


Table S3: MD simulation results of the VLE densities of pure $\mathrm{CO}_{2}$ from two different force fields (i.e. FF: Vrabec, FF: Werth)

\begin{tabular}{|l|l|l|l|l|}
\hline$T / \mathrm{K}$ & $\begin{array}{l}\rho_{\text {liq }} / \mathrm{kg} / \mathrm{m}^{3} \\
\text { FF: Vrabec }\end{array}$ & $\begin{array}{l}\rho_{\text {liq }} / \mathrm{kg} / \mathrm{m}^{3} \\
\text { FF: Werth }\end{array}$ & $\begin{array}{l}\rho_{\text {vap }} / \mathrm{kg} / \mathrm{m}^{3} \\
\text { FF: Vrabec }\end{array}$ & $\begin{array}{l}\rho_{\text {vap }} / \mathrm{kg} / \mathrm{m}^{3} \\
\text { FF: Werth }\end{array}$ \\
\hline 220 & 1143 & 1152 & 16 & 19 \\
\hline 250 & 1024 & 1011 & 48 & 57 \\
\hline 263 & 974 & 954 & 72 & 89 \\
\hline 273 & 920 & 888 & 98 & 127 \\
\hline 300 & 666 & & 300 & \\
\hline
\end{tabular}

Table S4: MD simulation results of pressure and surface tension of pure $\mathrm{CO}_{2}$ from two different force fields (i.e. FF: Vrabec, FF: Werth). Reported uncertainties for the surface tension are on a $95 \%$ confidence level.

\begin{tabular}{|l|l|l|l|l|}
\hline$T / \mathrm{K}$ & $\begin{array}{l}p / \mathrm{MPa} \\
\text { FF: Vrabec }\end{array}$ & $\begin{array}{l}p / \mathrm{MPa} \\
\text { FF: Werth }\end{array}$ & $\begin{array}{l}\gamma / \mathrm{mN} / \mathrm{m} \\
\text { FF: Vrabec }\end{array}$ & $\begin{array}{l}\gamma / \mathrm{mN} / \mathrm{m} \\
\text { FF: Werth }\end{array}$ \\
\hline 220 & 4.0 & 5.3 & $17.0 \pm 0.2$ & $16.5 \pm 0.2$ \\
\hline 250 & 16.4 & 19.7 & $8.0 \pm 0.2$ & $8.7 \pm 0.2$ \\
\hline 263 & 26.0 & 31.2 & $6.0 \pm 0.2$ & $5.7 \pm 0.2$ \\
\hline 273 & 34.8 & 41.4 & $4.0 \pm 0.2$ & $4.0 \pm 0.2$ \\
\hline
\end{tabular}


Table S5: Simulation results of surface tension over pressure of the $\mathrm{CO} 2 / \mathrm{CH} 4$ mixture

\begin{tabular}{|l|l|l|l|}
\hline \multicolumn{2}{|l|}{$T=223 \mathrm{~K}$} & $T=233 \mathrm{~K}$ \\
\hline$p / \mathrm{MPa}$ & $\gamma / \mathrm{mN} / \mathrm{m}$ & $p / \mathrm{MPa}$ & $\gamma / \mathrm{mN} / \mathrm{m}$ \\
\hline 2.37 & 10.7 & 2.32 & 10.4 \\
\hline 2.76 & 9.8 & 2.91 & 8.7 \\
\hline 3.28 & 7.9 & 3.38 & 7.6 \\
\hline 3.43 & 7.2 & 3.85 & 6.3 \\
\hline 3.63 & 6.5 & 4.19 & 5.2 \\
\hline 3.63 & 6.7 & 4.60 & 4.7 \\
\hline 3.97 & 5.9 & 4.86 & 3.9 \\
\hline 4.28 & 4.7 & & \\
\hline 4.45 & 4.1 & & \\
\hline
\end{tabular}


Table S6: MD simulation results of the VLE densities of the $\mathrm{CO}_{2} / \mathrm{CH}_{4}$ mixture

\begin{tabular}{|l|l|l|l|l|l|}
\hline \multicolumn{3}{|l}{$T=223 \mathrm{~K}$} & \multicolumn{3}{l|}{$T=233 \mathrm{~K}$} \\
\hline$p / \mathrm{MPa}$ & $\rho_{\text {liq }} / \mathrm{kg} / \mathrm{m}^{3}$ & $\rho_{\text {vap }} / \mathrm{kg} / \mathrm{m}^{3}$ & $p / \mathrm{MPa}$ & $\rho_{\text {liq }} / \mathrm{kg} / \mathrm{m}^{3}$ & $\rho_{\text {vap }} / \mathrm{kg} / \mathrm{m}^{3}$ \\
\hline 2.37 & 1049.0 & 41.5 & 2.32 & 1042.4 & 45.7 \\
\hline 2.76 & 1030.7 & 46.5 & 2.91 & 1002.9 & 54.5 \\
\hline 3.28 & 979.7 & 55.7 & 3.38 & 970.8 & 62.7 \\
\hline 3.43 & 965.7 & 60.3 & 3.85 & 932.3 & 71.1 \\
\hline 3.63 & 942.3 & 64.6 & 4.19 & 891.4 & 77.6 \\
\hline 3.63 & 941.5 & 62.9 & 4.60 & 856.5 & 87.0 \\
\hline 3.97 & 907.3 & 70.6 & 4.86 & 845.0 & 93.4 \\
\hline 4.45 & 892.1 & 73.8 & & & \\
\hline 4.28 & 879.2 & 75.1 & & & \\
\hline
\end{tabular}


Table S7: MD simulation results of the VLE Molar fractions of $\mathrm{CH}_{4}$ in the $\mathrm{CO}_{2} / \mathrm{CH}_{4}$ mixture

\begin{tabular}{|c|c|c|c|c|c|}
\hline \multicolumn{3}{|c|}{$T=223 \mathrm{~K}$} & \multicolumn{3}{|c|}{$T=233 \mathrm{~K}$} \\
\hline$p / \mathrm{MPa}$ & $\begin{array}{l}x_{C H 4, \text { liq }} \\
\mathrm{mol} / \mathrm{mol}\end{array}$ & $\begin{array}{l}x_{C H 4, \text { vap }} \\
\mathrm{mol} / \mathrm{mol}\end{array}$ & $p / \mathrm{MPa}$ & $\begin{array}{l}x_{\mathrm{CH} 4, \mathrm{liq}} \\
\mathrm{mol} / \mathrm{mol}\end{array}$ & $\begin{array}{l}x_{C H 4, v a p} \\
\mathrm{~mol} / \mathrm{mol}\end{array}$ \\
\hline 2.37 & 0.086 & 0.649 & 2.32 & 0.058 & 0.486 \\
\hline 2.76 & 0.101 & 0.677 & 2.91 & 0.091 & 0.564 \\
\hline 3.28 & 0.145 & 0.725 & 3.38 & 0.118 & 0.615 \\
\hline 3.43 & 0.157 & 0.722 & 3.85 & 0.150 & 0.647 \\
\hline 3.63 & 0.177 & 0.744 & 4.19 & 0.184 & 0.672 \\
\hline 3.63 & 0.177 & 0.742 & 4.60 & 0.214 & 0.686 \\
\hline 3.97 & 0.206 & 0.754 & 4.86 & 0.225 & 0.694 \\
\hline 4.45 & 0.220 & 0.768 & & & \\
\hline 4.28 & 0.231 & 0.778 & & & \\
\hline
\end{tabular}

